\title{
Ready for the design of voting rules?
}

\author{
Sascha Kurz*
}

October 15, 2018

\begin{abstract}
The design of fair voting rules has been addressed quite often in the literature. Still, the so-called inverse problem is not entirely resolved. We summarize some achievements in this direction and formulate explicit open questions and conjectures.

Keywords: voting power, power indices, inverse problem, design of voting rules, fair weights

JEL: C71, D71
\end{abstract}

\section{Introduction}

Voting in committees with dissimilar committee members raises the question of measuring individual power, i.e., the capability to influence a group decision under a given voting rule. To this end, power indices were introduced in order to quantify these abilities. Despite the fact that the question for the measurement of power dates back to the late 18th century, at the very least, there is still a very active research on power indices. Indeed we believe that this will also be the case for the next decades, giving a personal answer to a question about the future of power indices raised by Manfred Holler after finishing the collection [Holler \& Nurmi, 2013]. Its predecessor [Holler, 1982] contains the starting point of a vital and still widely open question in the area of power indices. To the best of our knowledge, Nurmi, 1982 was one of the first papers addressing the nowadays so-called inverse (power index) problem: Suppose one has a rather precise idea how the power, measured by a specific power index, should be distributed among the committee members - Penrose's square root law may serve as an example. How to design the voting rule (or mechanism) in order to meet the desired power distribution as closely as possible?

Of course partial answers have been given during the last 30 years, some of them quite recently, but we think that a lot remains to be explored. The aim of this article is to specify this and to propose explicit open questions and challenging conjectures.

${ }^{*}$ Department of Mathematics, University of Bayreuth, Germany. E-mail: sascha.kurz@unibayreuth.de. 


\section{Binary voting rules and the inverse problem}

A lot of models for the description of a voting situation in a committee have been proposed in the literature. Processes of opinion-formation, communication structures, a priori unions and dependencies have been considered. The decision space can be multi-dimensional, discrete or continuous. Here we want to stick to one of the most simple settings, the binary setting. Given a proposal, each voter can either say "yes" or "no", represented by 1 and 0 , respectively. The group decision is modeled as a simple game $v: 2^{N} \rightarrow\{0,1\}$ mapping the set of supporters to either a "yes"- or a "no"-decision (under some additional technical requirements). Weighted majority games are one of the most used binary voting rules in practice. Here each voter is assigned a non-negative weight $w_{i}$. If the sum of weights of the supporters meets or exceeds a given quota $q \in \mathbb{R}_{>0}$ the group decision is 1 and 0 otherwise.

Next we want to formalize the inverse power index problem. Let $\mathcal{C}$ be a class of voting rules, e.g., simple games or weighted majority games, for $n$ voters, $\|\cdot\|$ be a vector norm in $\mathbb{R}^{n}, \sigma \in \mathbb{R}_{\geq 0}^{n}$ be a vector of the desired power distribution, and $P: \mathcal{C} \rightarrow \mathbb{R}_{\geq 0}^{n}$ be a (positive) power index, e.g., the Shapley-Shubik index or the Banzhaf index. With this, the inverse problem asks for a minimizer $v^{\star}$ of $\|P(v)-\sigma\|$ over all $v \in \mathcal{C}$. In practice the determination of an approximate solution $v^{\prime}$, i.e., where the deviation $\left\|P\left(v^{\prime}\right)-\sigma\right\|$ is not too much larger as the minimum possible value $\left\|P\left(v^{\star}\right)-\sigma\right\|$, is also sufficient.

Weighted majority games can be designed by choosing an appropriate quota and appropriate weights. Taking the values of the desired power distribution $\sigma$ as weights $w$ and a quota of $q=\frac{1}{2}$, for example (or some suitable function of the weights), works quite well in some cases, while it does not in others, as we will see in the next section.

\section{Limit results}

In its strongest form, the so-called Penrose limit theorem states that, under certain technical conditions, the limit of the fraction of the power of two players equals the limit of the fraction of the respective weights. To make things precise, let $W \subseteq \mathbb{R}_{\geq 0}$ be a set of weights, $N^{(0)} \subsetneq N^{(1)} \subsetneq \ldots$ be an infinite increasing chain of finite non-empty sets, and $N=\bigcup_{n=0}^{\infty} N^{(n)}$. Each voter $i \in N$ is assigned a weight $w_{i} \in W$. With a fixed real number $q \in(0,1)$, we denote by $\mathcal{W}^{(n)}$ the weighted majority game with voter set $N^{(n)}$, weights $w_{i}$ for all $i \in N^{(n)}$ and quota $q \cdot \sum_{i \in N^{(n)}} w_{i}$. Given a power index $P$ we say that Penrose's limit theorem (PLT) holds for such a chain $\left(\mathcal{W}^{(n)}\right)_{n=0}^{\infty}$, if

$$
\lim _{n \rightarrow \infty} \frac{P_{i}\left(\mathcal{W}^{(n)}\right)}{P_{j}\left(\mathcal{W}^{(n)}\right)}=\frac{w_{i}}{w_{j}}
$$

for every pair of voters $i, j \in N$ with $w_{i}, w_{j} \neq 0$. We call a player $i$ regular if there exist constants $n_{0} \in \mathbb{N}$ and $\varepsilon>0$ such that

$$
\left|\left\{h \in N^{(n)} \mid w_{h}=w_{i}\right\}\right| \cdot \frac{w_{i}}{\sum_{h \in N^{(n)}} w_{h}} \geq \varepsilon
$$


for all $n \geq n_{0}$, i.e., the relative weight of all voters having the same weight as voter $i$ does not vanish. We call a chain non-atomic (or oceanic), if $\lim _{n \rightarrow \infty} w_{i} / \sum_{h \in N^{(n)}} w_{h}=$ 0 for all $i \in N$, i.e., if each individual weight vanishes.

In [Lindner \& Machover, 2004] the authors have proven that PLT holds for regular players in non-atomic chains for the Shapley-Shubik index with arbitrary relative quota $q$ and for the Banzhaf index with relative quota $q=\frac{1}{2}$.

Conjecture 1 PLT holds for regular players in non-atomic chains for the Banzhaf index with arbitrary relative quota $q \in(0,1)$.

In Kurz et al., 2014 it is shown that the corresponding statement for the nucleolus as a power index is true.

\section{Research question 2 For which power indices does Conjecture 1 hold?}

Looking at the example with one voter of weight 1 and all other voters having weight 2 , we see that, for symmetric power indices, the regularity, of at least the involved voters $i$ and $j$, is necessary. This subtlety, caused by divisibility properties of integers, can be circumvented by replacing the relative limit (1) with

$$
\lim _{n \rightarrow \infty} \sum_{i \in N^{(n)}}\left|P_{i}\left(\mathcal{W}^{(n)}\right)-w_{i}\right|=0
$$

i.e., the limit of the norm-1-difference between weights and power tends to zero.

Given such a limit result for an arbitrary power index, one can conclude the PLT result for regular players $i$ and $j$ in non-atomic chains. In Kurz et al., 2014 , Proposition 1] the underlying general proof strategy was applied to obtain the PLT result for the nucleolus. For the Shapley-Shubik index and non-atomic chains limit equation (3) was shown in Neyman, 1982.

Research question 3 For which power indices does limit equation (3) hold for non-atomic chains?

From a practical point of view, the big drawback of those results is the assumption of an infinite chain of weighted majority games. To obtain effective bounds for finite games a bit more is needed. And indeed the limit result for the nucleolus Nuc is based on the inequality

$$
\left\|\operatorname{Nuc}\left(\left[q ; w_{1}, \ldots, w_{n}\right]\right)-\left(w_{1}, \ldots, w_{n}\right)\right\|_{1} \leq \frac{2 \Delta}{\min (q, 1-q)},
$$

where $\Delta=\max _{1 \leq i \leq n} w_{i}, q \in(0,1), w \in \mathbb{R}_{\geq 0}$, and $\|w\|_{1}=1$, i.e. where $\Delta$ is the maximum weight assuming a normalized representation. We remark that $\Delta$ tends to zero in the non-atomic case.

Research question 4 For which power indices do constants $\alpha, \beta, c \in \mathbb{R}_{>0}$ exist, such that

$$
\left\|P\left(\left[q ; w_{1}, \ldots, w_{n}\right]\right)-\left(w_{1}, \ldots, w_{n}\right)\right\|_{1} \leq \frac{c \cdot \Delta^{\alpha}}{\min (q, 1-q)^{\beta}}
$$

holds for all $n \in \mathbb{N}, q \in(0,1), w \in \mathbb{R}_{\geq 0}$ with $\|w\|_{1}=1$ and $\Delta=\max _{1 \leq i \leq n} w_{i}$ ? 
For the Shapley-Shubik index such a bound may implicitly be contained in the technical results of [Neyman, 1982].

Conjecture 5 The answer to research question 4 is negative for the Public Good Index.

Of course we are not only interested in an existence result for such constants, but want to know them explicitly (and best possible).

\section{Conjecture 6}

$$
\left\|\operatorname{Nuc}\left(\left[q ; w_{1}, \ldots, w_{n}\right]\right)-\left(w_{1}, \ldots, w_{n}\right)\right\|_{1} \leq \frac{\Delta}{\min (q, 1-q)}
$$

is valid and tight for normalized weights.

So, given such a result and a specific desired power distribution $\sigma$, we can compute an upper bound for the norm-1-error between $\sigma$ as relative weights and the resulting power vector without evaluating the power index. This upper bound may also serve as a termination criterion for heuristic search algorithms for the inverse problem.

We can also obtain general assertions about the quality of the solution of taking power as weights in the inverse problem. If the maximum weight is not too far away from the average weight, i.e., if there exists a constant $C$ such that relative weights are bounded from above by $C \cdot \frac{1}{n}$, then the norm-1-error is in $O\left(\frac{1}{n}\right)$ (for $\alpha=1$ ), i.e., it decreases at least linearly in the number of voters.

The other case, where $\Delta$ does not tend to zero, i.e., if so-called atomic voters with non-vanishing weights are present, is also studied in the literature. Given a chain, let us call voters, whose relative weights can be bounded from below by a positive constant $\delta>0$, atomic, and those, whose relative weights tend to zero, oceanic. We assume that all voters are either atomic or oceanic and that the set $A$ of atomic players is finite. In Shapiro \& Shapley, 1978 it is proven (cf. Inequality 4.1) that

$$
\left|\operatorname{SSI}_{i}\left(\mathcal{W}^{(n)}\right)-\operatorname{SSI}_{i}\left(\mathcal{W}^{(m)}\right)\right| \in O\left(\frac{1}{n}\right)
$$

for all atomic players $i, n<m$, assuming that the relative weight of the oceanic players does not tend too slowly to zero. A precise formula for the limit of the Shapley-Shubik power of an atomic player is stated too. Similar results, while being a bit weaker, have been obtained for the Banzhaf index in Dubey \& Shapley, 1979.

Research question 7 For which power indices does the limit

$$
\lim _{n \rightarrow \infty} P_{i}\left(\mathcal{W}^{(n)}\right)
$$

exist for all atomic players $i \in A$, where $A$ is finite and the relative weights of the non-atomic players can be upper bounded by $C \cdot \frac{1}{n}$ with a constant $C>0$ ?

Conjecture 8 The nucleolus is a positive answer to research question 7 . 
Research question 9 Determine exact formulas for the positive answers to research question 7 .

For the nucleolus some first results in that direction are obtained in Galil, 1974.

\section{Distribution of power vectors}

Assuming $\sigma \in \mathbb{R}_{\geq 0}^{n}$ with $\|\sigma\|_{1}=1$, the space of the possible desired power distributions is an $n$-1-dimensional unit simplex. Since the number of weighted majority games or simple games, is finite, not every desired power vector can be met exactly. Using asymptotic bounds on the number of games one can conclude a lower bound for a worst case approximation error, which still tends to zero with increasing $n$, see Kurz \& Napel, 2014].

Efficient and positive power indices principally map into the $n$-1-dimensional unit simplex, so that the question arises whether for large $n$ each desired power vector can be approximated suitably well by the power vector of a weighted majority or a simple game. Some of the lesser known power indices satisfy $\frac{1}{c} \cdot \frac{1}{n} \leq P_{i}(v) \leq c \cdot \frac{1}{n}$ for all players $i$ and all simple games $v$, where $c>0$ is a constant independent from $n$. The Public Help index, see Bertini et al., 2008, is such an example; more can be found in Kurz, 2014. As a consequence only power distributions, where the entries do not differ too much, can be principally approximated well. For the extreme example $(1,0, \ldots, 0)$ we obtain a best possible approximation error tending to 2 , which is indeed the maximal distance between two non-negative vectors of sum 1 .

For the Banzhaf index such a constant $c$ does not exist. Nevertheless, e.g., for the power distribution $\sigma=(0.75,0.25,0, \ldots, 0)$ we have $\|\mathrm{Bz}(v)-\sigma\| \geq \frac{1}{9}$ for all simple games $n$, even if $n$ tends to infinity. This example, taken from Kurz, 2012b, is based on the seminal work of Alon \& Edelman, 2010:

Theorem 10 Let $n>k$ be positive integers, $\varepsilon<\frac{1}{k+1}$ be a positive real, and $v$ be a simple game on $n$ voters. If $\sum_{i=k+1}^{n} \mathrm{Bz}_{i}(v) \leq \varepsilon$, then there exists a simple game $v^{\prime}$ with $n-k$ dummies such that

$$
\left\|\mathrm{Bz}(v)-\mathrm{Bz}\left(v^{\prime}\right)\right\|_{1} \leq \frac{(2 k+1) \varepsilon}{1-(k+1) \varepsilon}+\varepsilon
$$

In other words the Banzhaf vector of a simple game, where most of the power is concentrated on just $k$ of the $n$ voters, has to be near, in an explicit sense, to the Banzhaf vector of another $k$-voter simple game.

In Kurz, 2014 such inequalities where called Alon-Edelman type results and indeed obtained for some other power indices besides the Banzhaf index.

Research question 11 For which power indices do Alon-Edelman type results do exist?

We remark that for the Johnston index, assuming a minor technical condition, no such result can exist. 
Conjecture 12 The nucleolus, the minimum sum representation index, the Shapley Shubik index and, more generally, p-binomial semivalues are positive answers to research question 11 .

For practical non-approximability computations the concrete bounds are of importance as well. The right hand side of (9) could indeed be decreased to $(2 k+2) \varepsilon$ in Kurz, 2014 and we ask for further improvements and worst case examples.

Conjecture 13 For $\sigma^{n}=(0.75,0.25,0, \ldots, 0) \in \mathbb{R}_{\geq 0}^{n}$ and for each simple game $v$ we have $\left\|\mathrm{Bz}(v)-\sigma^{n}\right\|_{1} \geq \frac{14}{37}$ and $\left\|\operatorname{SSI}(v)-\sigma^{n}\right\|_{1} \geq \frac{1}{3}$.

Conjecture 14 For each $\sigma \in \mathbb{R}_{\geq 0}^{n}$ with $\|\sigma\|_{1}=1$ and $n \geq 3$ there exists a weighted majority game $v$ with $\left\|\mathrm{S} \overline{\mathrm{SI}}(v)-\sigma^{n}\right\|_{1} \leq \frac{1}{3}$.

What about the nucleolus and other power indices? Are the worst-case examples unique? What happens when we consider only purely oceanic games?

With respect to the latter question, we consider the power distribution $\psi^{n}=$ $\frac{1}{2 n-1} \cdot(2, \ldots, 2,1)$. In Kurz \& Napel, 2014 the inequality

$$
\left\|P\left(\left[q ; \psi^{n}\right]\right)-\psi^{n}\right\|_{1} \geq \frac{2}{2 n-1} \cdot \frac{n-1}{n} \in \Theta\left(\frac{1}{n}\right)
$$

was shown for all symmetric, positive and efficient power indices $P$ for an arbitrary relative quota $q$. So for this special sequence of desired power distributions, taking the desired power vector as weights, yields an $\Omega(1 / n)$-error, which is still considerably large for medium sized constitutions like, e.g., $n=27$ or $n=28$.

Additionally, the authors solved the inverse power index problem for $\psi^{n}$ and the Banzhaf index. It turned out that within the class of weighted majority games, taking the optimal solution, the lower bound of (10) could be decreased by a small factor only. To the contrary, there exist exact solutions within the class of simple games for $6 \leq n \leq 18$ voters.

Conjecture 15 There exists a constant $c>0$ such that $\left\|\mathrm{Bz}(v)-\psi^{n}\right\|_{1} \geq \frac{c}{n}$ for all $n \in \mathbb{N}_{\geq 2}$ and all weighted majority games $v$.

Conjecture 16 For all $n \geq 6$ there exists a simple game $v$ such that $\operatorname{Bz}(v)=\psi^{n}$.

Research question 17 Does there exist a sequence of desired power distributions $\sigma^{n} \in \mathbb{R}_{\geq 0}^{n}$ with $\left\|\sigma^{n}\right\|_{1}=1$ and $\sigma_{i}^{n} \in O(1 / n)$ for all $1 \leq i \leq n$ such that $\left\|\mathrm{Bz}(v)-\sigma^{n}\right\|_{1} \in \Omega(1 / n)$ for all weighted majority (or simple) games $v$ ?

It seems that for the Banzhaf index there are some regions near the boundary of the unit simplex which can not be achieved as Banzhaf vectors of simple games. In the interior of the unit simplex the worst case distance to a Banzhaf vector of a weighted majority game is conjectured to be of order $\frac{1}{n}$.

Research question 18 Can we obtain more structural results on the set of achievable Banzhaf vectors? 
To make thinks more precise, we state a few of the known results. Let $\eta_{i}$ denote the number of swings of player $i$, i.e., the Banzhaf vector is proportional to the vector $\eta=\left(\eta_{1}, \ldots, \eta_{n}\right)$ of swings. The minimum and the maximum number of total swings, i.e., $n \leq\|\eta\|_{1} \leq\left(\left\lfloor\frac{n}{2}\right\rfloor+1\right) \cdot\left(\begin{array}{c}n \\ \left\lfloor\frac{n}{2}\right\rfloor+1\end{array}\right)$, are well known. Additionally, the $\eta_{i}$ are either all odd or all even numbers. If the underlying game is decisive, then the $\eta_{i}$ are all even and $\eta_{i}-\eta_{j}$ is divisible by 4, see e.g. [Dubey \& Shapley, 1979].

Of course the corresponding questions can be stated for other power indices too.

\section{$5 \quad$ Algorithmic approaches}

The first algorithms that were proposed for the inverse power index problem were heuristics (mainly hill-climbing and local-search), see e.g. Kurz \& Napel, 2014. Those heuristics are usually quite fast and produce small approximation errors on practical instances. Given the results from the previous section, a fundamental problem arises. Those methods do not provide a priori or a posteriori bounds on the achievable approximation error, which indeed may be large.

Conceptionally the easiest exact and general approach is based on exhaustive enumeration of a given class of voting games. For each game the power distribution, according to the chosen power index, and its corresponding distance to the desired power distribution can be computed easily. Taking the game with the smallest obtained distance solves the inverse problem exactly. Exhaustively generating weighted majority games, or one of its super classes, is not that hard, when the number of voters is rather small. For the class of so-called complete simple games there is a well known parameterization theorem, so that these games can be generated efficiently, see e.g. Kurz \& Tautenhahn, 2013. Since for $n \leq 7$ only few complete simple games are non-weighted, checking complete simple games for weightedness, see e.g. [Taylor \& Zwicker, 1999] for several such algorithms, is computationally feasible and was indeed done in the literature. For $n=8$ the fraction of weighted majority games within the class of complete simple games is roughly $\frac{1}{7}$ and roughly $\frac{1}{285}$ for $n=9$. Thus more direct algorithms for the enumeration of weighted majority games are needed. Notwithstanding the recent progress, see Kartak et al., 2014, de Keijzer et al., 2014, Kurz, 2012a, the number of weighted majority games is known up to $n=9$ voters only.

Research question 19 Determine the number of weighted majority and complete simple games for $n=10$ voters.

Due to the super exponential growth of both complete simple and weighted majority games we conjecture that the corresponding numbers for $n=11$ will not be determined exactly in the next decade. Since no intermediate lower bounds for approximability are computed, the application of exhaustive enumeration algorithms for the inverse problem is rather constrained.

Recently, algorithms for both the Banzhaf and the Shapley-Shubik index have been designed that can achieve a sufficiently accurate approximation with running polynomial time, in terms of the number of voters times a factor depending on the 
desired approximation quality, see De et al., 2012b, O'Donnell \& Servedio, 2011, De et al., 2012a.

Research question 20 Develop approximation algorithms for the inverse power index problem for other power indices besides the Banzhaf and the Shapley-Shubik index.

Besides the tailor-made approaches also integer linear programming techniques have been applied. For the Banzhaf and the Shapley-Shubik index an ILP formulation was stated in Kurz, 2012b. Describing an underlying counting structure of most of the known power indices, more such formulations were stated in Kurz, 2014.

Research question 21 Develop a computationally feasible integer linear programming approach for the inverse problem for the nucleolus and the minimum sum representation index.

Given the information which coalition of a simple or weighted majority game is winning in an ILP formulation, it is easy to state the Shapley-Shubik power of a voter by a precise formula for example. This is different for the two power indices mentioned in research question 21. In principle, the nucleolus can be described as the solution of a single but tremendously sized linear program, so that the dual linear program can be used to obtain such a formula within an ILP. See Freixas \& Kurz, 2014 for an application of this technique in the context of cooperative game theory. While we did not try this approach, we expect it to be computationally infeasible for rather small $n$.

Still the current optimization approaches for the inverse power index problem are limited to, say, $n=15$ voters, which is far beyond what can be achieved by exhaustive enumeration but not completely satisfactory.

Research question 22 Develop practical hybrid algorithms, which can solve the inverse power index problem routinely for up to, say, 30 voters, while giving reasonable a posteriori non-approximability bounds.

\section{Conclusion}

We have argued that a satisfactory solution of the inverse power index problem needs further progress with respect to

(1) the so-called limit results, which are currently mainly known for a very few power indices only;

(2) our understanding of the distribution of the attainable power vectors within the unit simplex;

(3) algorithmic solution approaches.

Indeed a lot needs to be done.

Citing several contemporary papers, we have a well justified hope, that there will be further progress in that direction during the next decades. This adds to 
the revelation of properties of power indices. However, for the application and the understanding of the political process, the question for the most appropriate decision rules is even more important than the hunt for the right index.

In order to encourage research on the broader context of the inverse problem, we have listed several research questions and explicit conjectures, which deserve to be either proven or falsified.

To answer the question of the title, I indeed believe that we are ready to design voting games for the inverse problem in the binary setting, but still more research is needed.

\section{References}

[Alon \& Edelman, 2010] Alon, N., \& Edelman, P.H. 2010. The inverse Banzhaf problem. Soc. Choice Welf., 34(3), 371-377.

[Bertini et al., 2008] Bertini, C., Gambarelli, G., \& Stach, I. 2008. A public help index. Pages 83-98 of: Braham, M., \& Steffen, F. (eds), Power, freedom, and voting. Springer.

[De et al., 2012a] De, A., Diakonikolas, I., \& Servedio, R.A. 2012a. The inverse Shapley value problem. Pages 266-277 of: Automata, Languages, and Programming. Springer.

[De et al., 2012b] De, A., Diakonikolas, I., Feldman, V., \& Servedio, R.A. 2012b. Nearly optimal solutions for the chow parameters problem and low-weight approximation of halfspaces. Pages 729-746 of: Proceedings of the 44th symposium on Theory of Computing. STOC '12. New York, USA: ACM.

[de Keijzer et al., 2014] de Keijzer, B., Klos, T., \& Zhang, Y. 2014. Finding optimal solutions for voting game design problems. Journal of Artificial Intelligence Research (JAIR). to appear.

[Dubey \& Shapley, 1979] Dubey, P., \& Shapley, L.S. 1979. Mathematical properties of the Banzhaf power index. Math. Oper. Res., 4(2), 99-131.

[Freixas \& Kurz, 2014] Freixas, J., \& Kurz, S. 2014. On $\alpha$-roughly weighted games. Internat. J. Game Theory. (to appear).

[Galil, 1974] Galil, Z. 1974. The nucleolus in games with major and minor players. Internat. J. Game Theory, 3(3), 129-140.

[Holler, 1982] Holler, M.J. 1982. Power, voting, and voting power. Springer.

[Holler \& Nurmi, 2013] Holler, M.J., \& Nurmi, H. (eds). 2013. Power, voting, and voting power: 30 years after. Berlin: Springer. xvii, 762 p.

[Kartak et al., 2014] Kartak, V.M., Kurz, S., Ripatti, A.V., \& Scheithauer, G. 2014. Minimal proper non-IRUP instances of the one-dimensional cutting stock problem. preprint. 
[Kurz, 2012a] Kurz, S. 2012a. On minimum sum representations for weighted voting games. Annals of Operations Research, 196(1), 361-369.

[Kurz, 2012b] Kurz, S. 2012b. On the inverse power index problem. Optimization, 61(8), 989-1011.

[Kurz, 2014] Kurz, S. 2014. The inverse problem for power distributions in committees. arXiv preprint 1402.0988, $46 \mathrm{pp}$.

[Kurz \& Napel, 2014] Kurz, S., \& Napel, S. 2014. Heuristic and exact solutions to the inverse power index problem for small voting bodies. Ann. Oper. Res., 215(1), 137-163.

[Kurz \& Tautenhahn, 2013] Kurz, S., \& Tautenhahn, N. 2013. On Dedekind's problem for complete simple games. Internat. J. Game Theory, 42(2), 411437.

[Kurz et al., 2014] Kurz, S., Napel, S., \& Nohn, A. 2014. The nucleolus of large majority games. Econ. Lett., 123(2), 139-143.

[Lindner \& Machover, 2004] Lindner, I., \& Machover, M. 2004. LS Penrose's limit theorem: proof of some special cases. Math. Social Sci., 47(1), 37-49.

[Neyman, 1982] Neyman, A. 1982. Renewal theory for sampling without replacement. Ann. Prob., 464-481.

[Nurmi, 1982] Nurmi, H. 1982. The problem of the right distribution of voting power. Pages 203-212 of: Power, Voting, and Voting Power. Springer.

[O'Donnell \& Servedio, 2011] O'Donnell, R., \& Servedio, R.A. 2011. The chow parameters problem. SIAM J. Comput., 40(1), 165-199.

[Shapiro \& Shapley, 1978] Shapiro, N.Z., \& Shapley, L.S. 1978. Values of large games, I: A limit theorem. Math. Oper. Res., 3(1), 1-9.

[Taylor \& Zwicker, 1999] Taylor, A.D., \& Zwicker, W.S. 1999. Simple games. Desirability relations, trading, pseudoweightings. Princeton, New Jersey: Princeton University Press. 246 p. 